\title{
HER2-targeted DARPin MP0274
}

National Cancer Institute

\section{Source}

National Cancer Institute. HER2-targeted DARPin MP0274. NCI Thesaurus. Code C148491.

A proprietary, designed ankyrin repeat proteins (DARPin)-based agent targeting the tyrosine kinase receptor epidermal growth factor receptor 2 (HER2; ErbB2), with potential antineoplastic activity. Compared to antibodies, DARPins are small in size, have favorable pharmacokinetics and allow for both high affinity binding and efficacy. Upon administration, the HER2-targeted DARPin MP0274 binds to two distinct nonoverlapping epitopes on HER2, thereby inhibiting the activity of HER2 and promoting HER2 internalization. This prevents HER2-mediated signaling, induces apoptosis and inhibits the growth of HER2-overexpressing tumor cells. DARPin also binds to human serum albumin, which extends the half-life of MP0274. HER2 is overexpressed in a variety of cancer cell types and is associated with increased tumor cell proliferation. 